\title{
New Interspecific Brassica Hybrids with High Levels of Heterosis for Fatty Acids Composition
}

\author{
Janetta Niemann ${ }^{1, * \mathbb{D}}$, Jan Bocianowski ${ }^{2} \mathbb{D}$, Kinga Stuper-Szablewska ${ }^{3}$ and \\ Tomasz Wojciechowski ${ }^{4}$ (D)
}

1 Department of Genetics and Plant Breeding, Faculty of Agronomy and Bioengineering, Poznań University of Life Sciences, Dojazd 11, 60-632 Poznań, Poland

2 Department of Mathematical and Statistical Methods, Faculty of Agronomy and Bioengineering, Poznań University of Life Sciences, Wojska Polskiego 28, 60-637 Poznań, Poland; jan.bocianowski@up.poznan.pl

3 Department of Chemistry, Faculty of Wood Technology, Poznań University of Life Sciences, Wojska Polskiego 75, 60-625 Poznań, Poland; kinga.stuper@up.poznan.pl

4 Institute of Biosystems Engineering, Faculty of Agronomy and Bioengineering, Poznań University of Life Sciences, Wojska Polskiego 50,60-627 Poznań, Poland; tomasz.wojciechowski@up.poznan.pl

* Correspondence: janetta.niemann@up.poznan.pl

Received: 5 May 2020; Accepted: 9 June 2020; Published: 10 June 2020

check for updates

\begin{abstract}
Winter oilseed rape (Brassica napus L.) is the most important oil crop in Europe. Optimizing the profile and quantity of fatty acids in rapeseed is critical for maximizing the value of edible oil. Although the utilization of crop heterosis for hybrid breeding in rapeseed is limited by the relatively narrow genetic basis of adapted germplasm, an up-to-date significant effort has been made to broaden the rapeseed gene pool using different strategies. The present study was aimed to estimate heterosis for oil quality of the newly developed Brassica interspecific hybrids, using selected parental lines. For this purpose, five parental genotypes and twenty-two interspecific cross-derived Brassica lines were evaluated in a randomized complete block design with three replications in the Greater Poland region during 2009, 2010 and 2011. Generally, the variation among genotypes was evident for most of the tested fatty acids mean values, but the differences between genotypes were not always statistically significant when based on individual fatty acids (FAs). However, the highest number of significant heterosis effects was observed for behenic and lignoceric acids and for Brassica hybrid line H1. Based on obtained results it was possible to select one genotype-the hybrid line $\mathrm{H} 5$, which is recommended for further inclusion in the breeding programs.
\end{abstract}

Keywords: Brassica hybrids; heterosis; fatty acids; gas chromatography

\section{Introduction}

Heterosis (hybrid vigor) has been successfully utilized in order to increase the productivity in several crop species such as maize, sugar beet, sunflower, forage crops and grasses. Several authors reported that hybrid vigor is evident also in seed yield of $F_{1}$ hybrids for both winter and summer types of oilseed rape B. napus [1,2]. Although, in Brassica oleracea, heterosis is the most efficient tool providing stimulus to the hybrid vegetable industry, in oilseed rape, heterosis has not been extensively exploited, because of the lack of an effective pollination control system for commercial production of $\mathrm{F}_{1}$ hybrid seeds $[3,4]$.

Rapeseed is considered as an important source of oil for industrial use, as well as for edible purposes. The objective of modifying oil quality is to develop oils with enhanced nutritional and functional properties. The industrial utilization of rapeseed oil requires a specific fatty acid composition, 
e.g., a high oleic acid content $[5,6]$. This type of fatty acid composition is also of interest as a cooking oil. The range of genetic variability for oleic acid content was thus greatly extended, which created the possibility for selection. The change of the fatty acid composition in interspecific crosses between different Brassica species is commonly known [7].

Fatty acids profile plays a key role in the use of Brassica oil by humans. Vegetable oilseed because of higher proportion of 16 and 18 carbons unsaturated fatty acids, mostly monounsaturated fatty acids (MUFAs) are utilized principally as a source of edible oil [8-10]. For this purpose, for example, linolenic acid (C18:3) is undesirable, because of reducing the durability of the oil, although it is an essential dietary fatty acid. Moreover, erucic acid (C22:1) contains nearly 50\% of total fatty acid, which is undesirable for human consumption, as it is reported to lead to myocardial lipidosis [11]. Although natural forms of rapeseed and mustard contain high levels of erucic acid (over $40 \%$ of total fatty acids), levels in rapeseed cultivated for food use are typically below $0.5 \%$.

Heterosis is agronomically important in the use of $F_{1}$ hybrid cultivars in many crops and vegetables, especially due to the superior performance, which can appear as biomass, yield and abiotic and biotic stress tolerance.

For obtaining a new hybrid plant, as a first step, some information on the genetic background of important characters should be collected. Next, these details are used to combine desirable traits in a hybrid. Therefore, especially for plant breeders it is essential to have detailed information about the desirable parental combination in hybridization programs, which usually resulted in a high degree of heterotic response. Furthermore, although interspecific hybrid lines do not meet the elite rapeseed standards, they are a valuable source for hybrid breeding due to their large distance from present breeding material and their high heterosis when combined with European rapeseed cultivars. Most heterosis research has been focused on increasing various quality traits in Brassica and other crops [12]. According to Pal and Sikka [13] heterosis is a quick, cheap and easy method for increasing crop production. To improve quality and quantity traits in Brassica and other crops, heterosis is one of the most effective methods. Owing to heterosis, breeders can increase plant production in a short time by utilization of less input [13].

Therefore, in the present study, an effort was made to identify promising Brassica genotypes to be used as a donor parent for the production of low erucic acid canola lines.

In the present studies, heterosis (better-parent) was estimated for the fatty acid composition in $\mathrm{F}_{1}-\mathrm{F}_{3}$ generations of Brassica interspecific hybrids, using the five parent cross experiment.

\section{Materials and Methods}

\subsection{Plant Material}

Plant material for the fatty acids composition analysis consisted of 27 Brassica genotypes including five parental genotypes, i.e., Brassica rapa ssp. pekinensis, Brassica rapa ssp. trilocularis cv. Yellow Sarson, B. carinata, B. juncea and the male sterile line of an $\mathrm{F}_{8}$ generation of B. napus (MS8), as well as twenty two interspecific cross-derived Brassica lines. The MS8 line was selected from resynthesized oilseed rape (B. rapa ssp. chinensis $\times$ B. oleracea var. gemmifera) using in vitro cultures of isolated embryos [14] (Table 1). $F_{1}-F_{3}$ generations of tested lines, as well as parental genotypes, were selected from the rapeseed breeding program of the Department of Genetics and Plant Breeding, Poznań University of Life Sciences (PULS).

Brassica genotypes were tested in the 2009, 2010 and 2011 growing seasons. Field trials were performed in three replicates of a randomized block design at the PULS experimental station Dłon (51 $41^{\prime} 23^{\prime \prime}$ N, $17^{\circ} 04^{\prime} 10^{\prime \prime}$ E) located $100 \mathrm{~km}$ south of Poznań, (Greater Poland, Poland). The field experiment in Dłoń was conducted on typical heavy soil of III quality class of good rye complex [14]. The sums of precipitation during the vegetation season of Brassica genotypes in 2009, 2010 and 2011 were respectively: $605.1,753.9$ and $465.5 \mathrm{~mm}$, but the mean temperatures during the vegetation season were respectively, $9.1,7.9$ and $9.8^{\circ} \mathrm{C}$. As shown in Table 2, these three seasons differed slightly in their 
climatic conditions. The field trials were arranged in a randomized complete block design with three replicates. Sowing dates were between the 24th and 28th of August. Each genotype was grown in a three-row plot of $9.0 \mathrm{~m}^{2}$, with a 0.30 row distance and a sawing density of 60 seeds $/ \mathrm{m}^{2}$.

Table 1. List of Brassica species and interspecific hybrids used as a research material.

\begin{tabular}{|c|c|c|c|}
\hline Genotype's Code & Species or Cross Combination & $\begin{array}{c}\text { Genotype's } \\
\text { Code }\end{array}$ & Species or Cross Combination \\
\hline S1 & Brassica napus MS8 line & & \\
\hline S2 & Brassica carinata & & \\
\hline S3 & Brassica juncea & & \\
\hline S4 & Brassica rapa ssp. pekinensis & & \\
\hline S5 & Brassica rapa ssp. trilocularis cv. Yellow Sarson & & \\
\hline H1 & B. napus $\times$ B. carinata $(125 / 1)$ & H12 & B. napus $\times$ Brassica rapa ssp. pekinensis $(14 / 1)$ \\
\hline $\mathrm{H} 2$ & B. napus $\times$ B. carinata $(125 / 2)$ & H13 & B. napus $\times$ Brassica rapa ssp. pekinensis $(18 / 1)$ \\
\hline $\mathrm{H} 3$ & B. napus $\times$ B. carinata $(126 / 1)$ & H14 & B. napus $\times$ Brassica rapa ssp. pekinensis $(19 / 1)$ \\
\hline $\mathrm{H} 4$ & B. napus $\times$ B. carinata $(126 / 2)$ & H15 & B. napus $\times$ Brassica rapa ssp. pekinensis $(20 / 1)$ \\
\hline H5 & B. napus $\times$ B. carinata $(127 / 2)$ & H16 & B. napus $\times$ Brassica rapa ssp. pekinensis $(24 / 1)$ \\
\hline $\mathrm{H} 6$ & B. napus $\times$ B. juncea $(55 / 1)$ & H17 & B. napus $\times$ Brassica rapa ssp. trilocularis (2) \\
\hline $\mathrm{H} 7$ & B. napus $\times$ B. juncea $(58 / 1)$ & $\mathrm{H} 18$ & B. napus $\times$ Brassica rapa ssp. trilocularis (5) \\
\hline $\mathrm{H} 8$ & B. napus $\times$ Brassica rapa ssp. pekinensis $(7 / 1)$ & H19 & B. napus $\times$ Brassica rapa ssp. trilocularis (6) \\
\hline H9 & B. napus $\times$ Brassica rapa ssp. pekinensis $(9 / 1)$ & $\mathrm{H} 20$ & B. napus $\times$ Brassica rapa ssp. trilocularis (43) \\
\hline $\mathrm{H} 10$ & B. napus $\times$ Brassica rapa ssp. pekinensis (12/1) & $\mathrm{H} 21$ & B. napus $\times$ Brassica rapa ssp. trilocularis (46) \\
\hline H11 & B. napus × Brassica rapa ssp. pekinensis(13/1) & $\mathrm{H} 22$ & B. napus $\times$ Brassica rapa ssp. trilocularis $(47)$ \\
\hline
\end{tabular}

Table 2. Meteorological conditions in Dłoń, during the vegetation season of winter oilseed rape in 2009, 2010 and 2011.

\begin{tabular}{cccc}
\hline \multirow{2}{*}{ Basic Weather Parameters } & \multicolumn{3}{c}{ Dłoń } \\
\cline { 2 - 4 } & $\mathbf{2 0 0 9}$ & $\mathbf{2 0 1 0}$ & $\mathbf{2 0 1 1}$ \\
\hline Mean annual temperature $\left({ }^{\circ} \mathrm{C}\right)$ & 9.1 & 7.9 & 9.8 \\
Sum of precipitation $(\mathrm{mm})$ & 605.1 & 753.9 & 465.5 \\
\hline
\end{tabular}

\subsection{Seeds Sampling and Analysis of Fatty Acids Composition:}

For each genotype, 10 single seeds, sampled from three to five plants, were analyzed and the average data were put to statistical analysis. Seeds were harvested at physiological maturity (BBCH 89). Fatty acid content was expressed as per cent of total fatty acid content.

A gas chromatography-flame ionization detection (GC-FID) method was used for direct quantitative analysis of seventeen fatty acids in seeds of analyzed Brassica genotypes in the Department of Biochemistry and Food Analysis of PULS. All the measurements were performed using a chromatograph Hewlett-Packard model 5890 series II with a flame ionization detector (FID), equipped with HP INNOWAX capilarry columns $(30 \mathrm{~m} \times 0.32 \mathrm{~mm} \times 0.15 \mathrm{~mm})$ and a split-splitless injector (Agilent Technologies, Inc., Cheshire, UK). Analyses of fatty acids were done at three replicates, and the arithmetic average was adopted for the results.

Seeds from all the parental materials and hybrids were subjected to analyses of the chosen fatty acid composition (C18:1—oleic acid, C18:2-linoleic acid, C18:3-linolenic acid, C20:0—arachidic acid, C20:1—eicosenoic acid, C22:0— behenic acid, C22:1—erucic acid and C24:0—lignoceric acid). The fatty acid content of the seed oil was measured by gas chromatography of the fatty acid methylesters, according to the protocol described by Momotaz et al. (2000) [15].

\subsection{Statistical Analysis}

Analysis for parental forms and hybrids were made independently. Firstly, the normality of the distributions of the studied traits were tested using Shapiro-Wilk's normality test [16]. A multivariate analysis of variance (MANOVA) was performed. The canonical variate analysis was applied in order to present a multi-trait assessment of similarity for the tested genotypes (parental forms and/or hybrids) in a lower number of dimensions with the least possible loss of information [17]. This makes it possible to illustrate variation in genotypes in terms of all the observed traits in the graphic form. The Mahalanobis 
distance was suggested as a measure of "polytrait" genotypes similarity [18], the significance of which was verified by means of critical value $\mathrm{D}_{\alpha}$ called "the least significant distance" [19]. Mahalanobis distances were calculated. Next, the heterosis effects of all observed traits were tested for each year of study. All the analyses were conducted using the GenStat 18 statistical software package (VSN International Limited, Hemel Hempstead, HP2 4TP, UK).

\section{Results and Discussion}

Nowadays, selecting high quality rapeseed, with good yield and increased oil content and improved edible oils with a modified fatty acid composition, seems to be one of the most important B. napus breeding goals [20]. Furthermore, palmitic, stearic, oleic, linoleic, linolenic, eicosenoic and erucic acids are the most important fatty acids in rapeseed, which determines the flavor and nutritional quality of B. napus [21]. However, reports in the literature on the performance of B. napus interspecific hybrids, with respect to the potential of heterosis for seed quality traits in rapeseed, are relatively rare [22]. In order to improve breeding efficiency, the selection of desirable genotypes is based mostly on a selected trait, i.e., the fatty acid composition regarded as nutritionally favorable [23]. Therefore, the multivariate approach to evaluating the fatty acid composition of Brassica hybrids seed oil can be useful in the assessment and selection of genotypes with the fatty acid composition advantageous from the human nutrition point of view.

The conducted multivariate analysis of variance (MANOVA) made it possible to reject tested hypothesis concerning a lack of average multivariate differences between parental forms $(P<0.001)$. Mean values of eleven prominent fatty acids (FA) (16:0, 16:1, 18:0, 18:1, 18:2, 18:3, 20:0, 20:1, 22:0, 22:1 and 24:0) differ between parental forms (Figure 1, Table 3). Generally, the variation among genotypes was evident for most of the tested fatty acids mean values, but the differences between genotypes were not always statistically significant when based on individual FAs. The highest C22:1 mean value was observed in B. trilocularis (61.41\%), ranging from $13.10 \%$ in B. napus MS8 line to $61.41 \%$ in B. trilocularis, but the minimum $C 16: 1$ value was noticed $(0.17 \%)$ in B. carinata and B. juncea. Similarly, a low mean value of C24:0 was observed in MS8 line and in B. juncea. The most noticeable differences in the fatty acid composition between B. napus MS8 line seeds and other parental Brassica species were observed (Figure 1). For example, the erucic acid (C22:1) composition of B. napus and B. trilocularis seeds was $4.24 \%$ and $1.76 \%$ palmitic acid (C16:0), respectively, $1.69 \%$ and $1.03 \%$ stearic acid (C18:0), $42.33 \%$ and $12.1 \%$ oleic acid (C18:1), respectively, $17.56 \%$ and $9.8 \%$ linoleic acid (C18:2), $8.32 \%$ and $5.12 \%$ linolenic acid (C18:3), $11.59 \%$ and $4.96 \%$ eicosenoic acid (C20:1), and $13.1 \%$ and $61.41 \%$ erucic acid (C22:1).

Individual traits are of different importance and have a different share in the joint multivariate variation. A study on the multivariate variation for parental forms also includes an identification of the most important traits in the multivariate variation of parental forms. The analysis of canonical variables is a statistical tool making it possible to solve this problem [24-26]. Results of the analysis of canonical variables for investigated parental forms are presented in Figure 2. The first two canonical variables explained jointly $97.16 \%$ total variation between parental forms (Figure 2).

The greatest variation of parental forms in terms of all the traits jointly (measured Mahalanobis distances) was found for MS8 and B. carinata (the Mahalanobis distance between them amounted to 143.8). The greatest similarity was found for B. juncea and B. pekinensis (30.4). Mahalanobis distances between MS8 and other parental forms were statistically significant (Table 4). 
Table 3. Fatty acid profile (\% of total identified, mean values \pm SD) in analyzed Brassica genotypes.

\begin{tabular}{|c|c|c|c|c|c|c|c|c|c|c|c|c|c|c|c|c|c|c|c|c|c|c|}
\hline \multirow{2}{*}{ Genotypes } & \multicolumn{2}{|c|}{ C16:0 } & \multicolumn{2}{|c|}{ C16:1 } & \multicolumn{2}{|c|}{ C18:0 } & \multicolumn{2}{|c|}{ C18:1 } & \multicolumn{2}{|c|}{ C18:2 } & \multicolumn{2}{|c|}{ C18:3 } & \multicolumn{2}{|c|}{ C20:0 } & \multicolumn{2}{|c|}{ C20:1 } & \multicolumn{2}{|c|}{ C22:0 } & \multicolumn{2}{|c|}{ C22:1 } & \multicolumn{2}{|c|}{ C24:0 } \\
\hline & Mean & s.d. & Mean & s.d. & Mean & s.d. & Mean & s.d. & Mean & s.d. & Mean & s.d. & Mean & s.d. & Mean & s.d. & Mean & s.d. & Mean & s.d. & Mean & s.d. \\
\hline S1 & 4.24 & 0.10 & 0.202 & 0.008 & 1.692 & 0.134 & 42.34 & 0.37 & 17.68 & 0.86 & 8.46 & 0.50 & 0.692 & 0.044 & 11.6 & 0.32 & 0.39 & 0.03 & 13.1 & 0.50 & 0.168 & 0.04 \\
\hline S2 & 3.658 & 0.27 & 0.168 & 0.016 & 1.196 & 0.134 & 10.42 & 0.41 & 20.06 & 0.46 & 8.576 & 0.31 & 1.068 & 0.035 & 7.04 & 0.36 & 1.24 & 0.24 & 44.84 & 0.74 & 1.638 & 0.14 \\
\hline S3 & 3.626 & 0.27 & 0.172 & 0.043 & 1.27 & 0.210 & 16.12 & 0.13 & 19.26 & 0.72 & 17.306 & 1.81 & 0.66 & 0.207 & 6.2 & 0.14 & 0.048 & 0.01 & 35.08 & 1.84 & 0.188 & 0.11 \\
\hline S4 & 2.818 & 0.04 & 0.182 & 0.027 & 1.318 & 0.019 & 17.38 & 0.35 & 14.35 & 0.21 & 8.88 & 0.12 & 1.018 & 0.036 & 7.62 & 0.16 & 1.206 & 0.06 & 43.68 & 0.29 & 1.448 & 0.22 \\
\hline S5 & 1.758 & 0.15 & 0.152 & 0.008 & 1.028 & 0.011 & 12.1 & 0.08 & 9.8 & 0.12 & 5.116 & 0.06 & 1.106 & 0.032 & 4.95 & 0.08 & 1.296 & 0.06 & 61.44 & 0.28 & 0.988 & 0.02 \\
\hline H1 F1 & 4.556 & 0.36 & 0.216 & 0.017 & 1.612 & 0.106 & 39.2 & 3.06 & 14.9 & 1.15 & 7.246 & 1.15 & 0.75 & 0.043 & 13.18 & 0.69 & 0.412 & 0.03 & 17.61 & 1.71 & 0.2 & 0.04 \\
\hline H1 F2 & 4.482 & 0.17 & 0.43 & 0.020 & 1.74 & 0.046 & 36.96 & 1.37 & 15.78 & 1.12 & 8.348 & 0.56 & 0.424 & 0.015 & 12.85 & 0.95 & 0.096 & 0.04 & 18.63 & 0.90 & 0.094 & 0.01 \\
\hline H1 F3 & 4.519 & 0.17 & 0.323 & 0.016 & 1.676 & 0.044 & 38.08 & 1.30 & 15.34 & 0.75 & 7.797 & 0.46 & 0.581 & 0.019 & 13.01 & 0.71 & 0.255 & 0.03 & 18.11 & 0.94 & 0.155 & 0.03 \\
\hline $\mathrm{H} 2 \mathrm{~F} 1$ & 4.452 & 0.33 & 0.184 & 0.028 & 1.612 & 0.167 & 30.66 & 0.88 & 10.38 & 0.42 & 4.308 & 0.29 & 0.906 & 0.009 & 16.2 & 0.04 & 0.628 & 0.11 & 30.25 & 0.56 & 0.256 & 0.05 \\
\hline $\mathrm{H} 2 \mathrm{~F} 2$ & 4.488 & 0.45 & 0.202 & 0.019 & 1.694 & 0.073 & 30.76 & 1.06 & 11.82 & 0.96 & 3.944 & 0.46 & 0.902 & 0.016 & 15.97 & 0.14 & 0.578 & 0.04 & 29.2 & 0.14 & 0.218 & 0.03 \\
\hline H2 F3 & 4.458 & 0.39 & 0.194 & 0.018 & 1.653 & 0.108 & 30.86 & 0.72 & 11.03 & 0.89 & 4.133 & 0.13 & 0.905 & 0.007 & 16.25 & 0.18 & 0.603 & 0.06 & 29.47 & 0.21 & 0.238 & 0.03 \\
\hline H3 F1 & 4.26 & 0.19 & 0.184 & 0.009 & 1.494 & 0.070 & 33.22 & 0.82 & 13.57 & 0.65 & 6.524 & 0.76 & 0.748 & 0.041 & 15.71 & 0.19 & 0.446 & 0.01 & 23.52 & 0.53 & 0.224 & 0.03 \\
\hline H3 F2 & 4.284 & 0.19 & 0.128 & 0.099 & 1.504 & 0.085 & 33.08 & 0.46 & 13.46 & 0.50 & 6.372 & 0.49 & 0.972 & 0.018 & 16 & 0.33 & 0.45 & 0.03 & 23.37 & 0.44 & 0.238 & 0.02 \\
\hline H3 F3 & 4.272 & 0.19 & 0.157 & 0.046 & 1.5 & 0.076 & 33.07 & 0.58 & 13.51 & 0.57 & 6.508 & 0.53 & 0.86 & 0.026 & 15.79 & 0.07 & 0.454 & 0.01 & 23.52 & 0.46 & 0.232 & 0.03 \\
\hline H34 F1 & 4.53 & 0.05 & 0.198 & 0.008 & 1.608 & 0.019 & 36.58 & 0.34 & 13.84 & 0.20 & 6.666 & 0.16 & 0.732 & 0.004 & 14.44 & 0.05 & 0.382 & 0.03 & 20.69 & 0.29 & 0.226 & 0.05 \\
\hline H4 F2 & 4.482 & 0.20 & 0.142 & 0.111 & 1.586 & 0.110 & 35.29 & 1.98 & 13.74 & 0.37 & 6.632 & 0.23 & 0.972 & 0.018 & 15.26 & 1.21 & 0.402 & 0.03 & 21.1 & 1.00 & 0.232 & 0.02 \\
\hline H4 F3 & 4.506 & 0.13 & 0.171 & 0.058 & 1.598 & 0.061 & 35.8 & 0.94 & 13.78 & 0.26 & 6.638 & 0.19 & 0.854 & 0.009 & 15 & 0.37 & 0.392 & 0.03 & 0.9 & 0.66 & 0.229 & 0.03 \\
\hline H5 F1 & 5.888 & 0.27 & 0.246 & 0.015 & 2.16 & 0.046 & 59.67 & 3.19 & 10.18 & 1.63 & 3.382 & 0.89 & 0.83 & 0.037 & 8.81 & 0.40 & 0.43 & 0.03 & 8.04 & 0.61 & 0.252 & 0.12 \\
\hline H5 F2 & 5.77 & 0.36 & 0.238 & 0.013 & 2.636 & 0.319 & 59.48 & 1.73 & 10.41 & 1.00 & 3.394 & 0.62 & 0.768 & 0.094 & 8.61 & 0.45 & 0.376 & 0.06 & 7.95 & 0.56 & 0.218 & 0.10 \\
\hline $\mathrm{H} 5 \mathrm{H} 3$ & 5.826 & 0.22 & 0.242 & 0.013 & 2.334 & 0.187 & 59.7 & 2.15 & 10.11 & 1.07 & 3.394 & 0.56 & 0.799 & 0.034 & 8.73 & 0.37 & 0.404 & 0.03 & 8.08 & 0.44 & 0.234 & 0.08 \\
\hline H6 F1 & 3.626 & 0.27 & 0.172 & 0.043 & 1.27 & 0.210 & 19.01 & 2.16 & 13.1 & 0.59 & 13.726 & 0.34 & 0.66 & 0.207 & 6.56 & 0.54 & 0.048 & 0.01 & 41.58 & 0.98 & 0.176 & 0.08 \\
\hline H6 F2 & 3.678 & 0.35 & 0.278 & 0.092 & 1.314 & 0.255 & 16.77 & 0.39 & 13.92 & 0.53 & 13.07 & 0.47 & 1.386 & 0.246 & 7.43 & 0.27 & 0.44 & 0.11 & 40.79 & 0.40 & 0.886 & 0.37 \\
\hline H6 F3 & 3.652 & 0.12 & 0.225 & 0.063 & 1.292 & 0.223 & 16.76 & 0.51 & 13.58 & 0.25 & 14.394 & 0.83 & 1.029 & 0.157 & 6.93 & 0.31 & 0.243 & 0.05 & 41.33 & 0.28 & 0.531 & 0.19 \\
\hline H7 F1 & 4.208 & 0.15 & 0.228 & 0.036 & 1.582 & 0.111 & 61.31 & 0.85 & 18.36 & 0.73 & 9.018 & 0.44 & 0.628 & 0.029 & 2.88 & 0.37 & 0.418 & 0.04 & 1.19 & 0.19 & 0.096 & 0.04 \\
\hline H7 F2 & 4.112 & 0.15 & 0.332 & 0.084 & 1.802 & 0.619 & 60.39 & 0.65 & 18.1 & 0.34 & 8.922 & 0.19 & 0.866 & 0.505 & 3.78 & 0.31 & 0.432 & 0.14 & 1.01 & 0.12 & 0.2 & 0.11 \\
\hline H7 F3 & 4.065 & 0.31 & 037 & 0.234 & 1736 & 0.306 & 51.84 & 19.63 & 17.31 & 2.03 & 9.919 & 1.95 & 0.852 & 0.337 & 4.06 & 1.83 & 0.407 & 0.09 & 9.2 & 18.17 & 0.174 & 0.09 \\
\hline H8 F1 & 4.048 & 0.33 & 0.196 & 0.015 & 1.774 & 0.080 & 40.12 & 2.92 & 14.92 & 0.54 & 7.54 & 1.17 & 0.774 & 0.033 & 13.73 & 1.43 & 0.35 & 0.04 & 16.48 & 1.70 & 0.048 & 0.07 \\
\hline H8 F2 & 3.938 & 0.43 & 0.196 & 0.015 & 1.72 & 0.162 & 39.65 & 4.41 & 14.31 & 2.01 & 7.51 & 2.30 & 0.766 & 0.047 & 14.8 & 1.31 & 0.36 & 0.05 & 16.61 & 1.92 & 0.092 & 0.06 \\
\hline H8 F3 & 3.816 & 0.21 & 0.192 & 0.018 & 1.612 & 0.035 & 37.0 & 1.4 & 15.2 & 0.7 & 8.254 & 0.50 & 0.75 & 0.027 & 13.96 & 1.01 & 0.376 & 0.02 & 18.45 & 1.47 & 0.17 & 0.04 \\
\hline H9 F1 & 3.586 & 0.32 & 0.188 & 0.026 & 1.6 & 0.077 & 26.65 & 5.17 & 12.86 & 2.16 & 7.286 & 0.90 & 0.81 & 0.092 & 16.24 & 1.07 & 0.396 & 0.07 & 30.13 & 7.26 & 0.22 & 0.05 \\
\hline H9 F2 & 4.172 & 0.35 & 0.246 & 0.063 & 1.544 & 0.064 & 32.04 & 1.77 & 15.05 & 2.39 & 6.918 & 1.59 & 0.802 & 0.051 & 14.09 & 1.25 & 0.556 & 0.14 & 24.22 & 2.36 & 0.238 & 0.10 \\
\hline H9 F3 & 4.786 & 0.34 & 0.208 & 0.015 & 1.76 & 0.096 & 31.57 & 1.02 & 11.1 & 0.81 & 4.638 & 0.78 & 0.876 & 0.038 & 16.68 & 0.21 & 0.472 & 0.04 & 27.53 & 0.60 & 0.234 & 0.05 \\
\hline H10 F1 & 3.55 & 0.55 & 0.174 & 0.025 & 1.532 & 0.166 & 28.75 & 2.67 & 11.27 & 1.88 & 5.5 & 1.60 & 0.89 & 0.070 & 13.6 & 0.55 & 0.672 & 0.09 & 33.59 & 2.41 & 0.372 & 0.07 \\
\hline H10 F2 & 4.826 & 0.93 & 0.214 & 0.034 & 1.718 & 0.234 & 32.26 & 2.62 & 11.45 & 0.41 & 4.832 & 1.04 & 0.858 & 0.030 & 13.9 & 2.81 & 0.52 & 0.08 & 28.92 & 1.15 & 0.284 & 0.03 \\
\hline $\mathrm{H} 10 \mathrm{~F} 3$ & 5.038 & 0.67 & 0.22 & 0.014 & 1.928 & 0.266 & 35.15 & 3.07 & 10.79 & 3.51 & 5.806 & 3.63 & 0.928 & 0.110 & 14.57 & 1.76 & 0.548 & 0.03 & 24.45 & 1.68 & 0.218 & 0.07 \\
\hline H11 F1 & 4.156 & 0.62 & 0.192 & 0.024 & 1.85 & 0.197 & 46.91 & 5.54 & 15.16 & 2.34 & 7.794 & 2.51 & 0.768 & 0.119 & 11.58 & 2.17 & 0.37 & 0.10 & 11.06 & 1.71 & 0.116 & 0.17 \\
\hline H11 F2 & 4.316 & 0.29 & 0.21 & 0.024 & 1.932 & 0.123 & 57.98 & 3.16 & 17.47 & 1.16 & 7.714 & 0.84 & 0.754 & 0.053 & 5.1 & 1.81 & 0.388 & 0.06 & 4.04 & 1.74 & 0.036 & 0.08 \\
\hline H11 F3 & 4.302 & 0.48 & 0.198 & 0.038 & 1.918 & 0.223 & 61.05 & 3.26 & 19.82 & 1.75 & 8.258 & 1.07 & 0.734 & 0.101 & 2.26 & 0.41 & 0.404 & 0.06 & 1 & 0.48 & 0 & 0.00 \\
\hline
\end{tabular}


Table 3. Cont

\begin{tabular}{|c|c|c|c|c|c|c|c|c|c|c|c|c|c|c|c|c|c|c|c|c|c|c|}
\hline \multirow{2}{*}{ Genotypes } & \multicolumn{2}{|c|}{ C16:0 } & \multicolumn{2}{|c|}{ C16:1 } & \multicolumn{2}{|c|}{ C18:0 } & \multicolumn{2}{|c|}{ C18:1 } & \multicolumn{2}{|c|}{ C18:2 } & \multicolumn{2}{|c|}{ C18:3 } & \multicolumn{2}{|c|}{ C20:0 } & \multicolumn{2}{|c|}{ C20:1 } & \multicolumn{2}{|c|}{ C22:0 } & \multicolumn{2}{|c|}{ C22:1 } & \multicolumn{2}{|c|}{ C24:0 } \\
\hline & Mean & s.d. & Mean & s.d. & Mean & s.d. & Mean & s.d. & Mean & s.d. & Mean & s.d. & Mean & s.d. & Mean & s.d. & Mean & s.d. & Mean & s.d. & Mean & s.d. \\
\hline H12 F1 & 4.032 & 0.15 & 0.198 & 0.004 & 1.682 & 0.137 & 39.83 & 2.57 & 16.48 & 0.93 & 9.244 & 0.45 & 0.72 & 0.053 & 12.28 & 0.72 & 0.358 & 0.02 & 15 & 1.91 & 0.158 & 0.04 \\
\hline $\mathrm{H} 12 \mathrm{~F} 2$ & 4.246 & 0.53 & 0.204 & 0.015 & 1.96 & 0.238 & 47.58 & 2.93 & 15.31 & 1.77 & 7.164 & 2.12 & 0.8 & 0.090 & 10.84 & 2.05 & 0.376 & 0.04 & 11.41 & 1.80 & 0.07 & 0.10 \\
\hline H12 F3 & 3.878 & 0.30 & 0.194 & 0.009 & 1.8 & 0.118 & 42.12 & 1.72 & 14.73 & 1.54 & 7.956 & 1.03 & 0.754 & 0.098 & 12.86 & 1.09 & 0.352 & 0.05 & 15.19 & 1.81 & 0.106 & 0.02 \\
\hline H13 F1 & 4.866 & 0.80 & 0.272 & 0.119 & 1.764 & 0.202 & 46.05 & 6.03 & 13.04 & 1.12 & 6.05 & 1.53 & 0.892 & 0.263 & 12.54 & 2.04 & 0.458 & 0.11 & 13.65 & 2.79 & 0.188 & 0.06 \\
\hline H13 F2 & 5.108 & 0.98 & 0.242 & 0.061 & 2.056 & 0.188 & 53.3 & 5.05 & 12.97 & 1.90 & 5.714 & 2.06 & 0.848 & 0.086 & 9.31 & 1.40 & 0.452 & 0.06 & 9.61 & 2.47 & 0.17 & 0.12 \\
\hline H13 F3 & 6.118 & 0.62 & 0.25 & 0.012 & 2.172 & 0.150 & 58.94 & 3.11 & 9.8 & 1.31 & 2.89 & 0.69 & 0.952 & 0.084 & 8.69 & 1.02 & 0.586 & 0.03 & 9.04 & 1.12 & 0.224 & 0.09 \\
\hline H14 F1 & 2.824 & 0.21 & 0.146 & 0.015 & 1.322 & 0.164 & 19.23 & 3.93 & 7.82 & 1.27 & 3.9 & 1.39 & 0.998 & 0.095 & 12.18 & 1.21 & 0.974 & 0.08 & 50.15 & 4.16 & 0.334 & 0.19 \\
\hline H14 F2 & 3.386 & 0.12 & 0.166 & 0.013 & 1.61 & 0.206 & 30.85 & 2.59 & 13.18 & 0.57 & 7.838 & 0.49 & 0.832 & 0.075 & 16.05 & 0.87 & 0.434 & 0.05 & 25.44 & 2.04 & 0.18 & 0.11 \\
\hline H14 F3 & 4.226 & 0.18 & 0.258 & 0.077 & 1.618 & 0.096 & 35.2 & 2.82 & 13.28 & 2.08 & 6.604 & 1.71 & 0.924 & 0.232 & 15.32 & 1.46 & 0.474 & 0.12 & 21.9 & 2.54 & 0.144 & 0.11 \\
\hline H15 F1 & 4.73 & 0.50 & 0.21 & 0.007 & 1.844 & 0.106 & 36.77 & 2.12 & 11.06 & 1.09 & 4.502 & 0.98 & 0.862 & 0.044 & 15.98 & 1.03 & 0.456 & 0.09 & 23.22 & 1.08 & 0.166 & 0.06 \\
\hline H15 F2 & 4.54 & 0.67 & 0.208 & 0.022 & 1.856 & 0.207 & 37.14 & 2.67 & 12.45 & 2.11 & 6.24 & 2.66 & 0.81 & 0.072 & 14.64 & 1.12 & 0.41 & 0.02 & 21.38 & 0.30 & 0.168 & 0.03 \\
\hline H15 F3 & 4.656 & 0.72 & 0.212 & 0.026 & 1.762 & 0.228 & 38.01 & 2.24 & 12.69 & 1.92 & 6.184 & 2.07 & 0.776 & 0.059 & 14.66 & 0.79 & 0.408 & 0.05 & 20.29 & 0.57 & 0.202 & 0.09 \\
\hline H16 F1 & 4.134 & 0.29 & 0.228 & 0.025 & 1.542 & 0.058 & 32.78 & 2.68 & 15.8 & 0.99 & 8.52 & 0.77 & 0.744 & 0.053 & 13.7 & 2.08 & 0.424 & 0.11 & 21.8 & 2.55 & 0.238 & 0.08 \\
\hline H16 F2 & 4.144 & 0.38 & 0.22 & 0.043 & 1.716 & 0.101 & 36.26 & 2.13 & 16.26 & 1.86 & 8.064 & 1.04 & 0.77 & 0.045 & 13.21 & 1.58 & 0.432 & 0.08 & 18.65 & 1.23 & 0.212 & 0.07 \\
\hline H16 F3 & 4.766 & 0.48 & 0.226 & 0.015 & 1.676 & 0.109 & 40.89 & 3.74 & 15.03 & 2.11 & 6.632 & 1.58 & 0.776 & 0.040 & 13.1 & 0.13 & 0.426 & 0.03 & 16.2 & 0.75 & 0.158 & 0.04 \\
\hline H17 F1 & 4.564 & 0.15 & 0.2 & 0.012 & 1.538 & 0.023 & 42.77 & 3.50 & 14.72 & 0.28 & 7.3 & 0.61 & 0.754 & 0.053 & 10.91 & 1.11 & 0.466 & 0.15 & 16.46 & 5.08 & 0.174 & 0.05 \\
\hline H17 F2 & 4.152 & 0.52 & 0.156 & 0.074 & 1.46 & 0.182 & 36.19 & 5.34 & 14.6 & 2.59 & 7.222 & 1.94 & 0.772 & 0.090 & 10.85 & 3.03 & 0.578 & 0.17 & 23.68 & 5.85 & 0.264 & 0.11 \\
\hline H17 F3 & 4.654 & 0.16 & 0.196 & 0.015 & 1.596 & 0.127 & 43.64 & 1.64 & 13.95 & 1.87 & 6.79 & 1.78 & 0.784 & 0.117 & 11.01 & 0.89 & 0.48 & 0.19 & 16.64 & 5.48 & 0.164 & 0.04 \\
\hline H18 F1 & 3.046 & 0.28 & 0.172 & 0.024 & 1.338 & 0.100 & 25.37 & 1.63 & 13.14 & 2.53 & 7.5 & 1.93 & 0.832 & 0.054 & 12.5 & 0.92 & 0.708 & 0.08 & 34.82 & 3.69 & 0.406 & 0.07 \\
\hline H18 F2 & 3.042 & 0.22 & 0.176 & 0.026 & 1.308 & 0.134 & 23.82 & 2.67 & 12.48 & 1.84 & 7.474 & 1.74 & 0.848 & 0.078 & 11.98 & 0.92 & 0.76 & 0.03 & 37.51 & 2.49 & 0.432 & 0.14 \\
\hline H18 F3 & 3.058 & 0.28 & 0.172 & 0.024 & 1.34 & 0.100 & 25.63 & 2.00 & 13.22 & 2.55 & 7.64 & 1.98 & 0.826 & 0.056 & 12.51 & 0.92 & 0.7 & 0.08 & 344 & 4.20 & 0.4 & 0.08 \\
\hline H19 F1 & 3.13 & 0.17 & 0.154 & 0.009 & 1.414 & 0.029 & 23.46 & 6.13 & 9.26 & 2.02 & 4.396 & 1.68 & 0.94 & 0.085 & 12.82 & 1.10 & 0.868 & 0.15 & 43.03 & 8.51 & 0.372 & 0.05 \\
\hline H19 F2 & 5.762 & 1.28 & 0.246 & 0.061 & 1.942 & 0.320 & 54.17 & 4.65 & 12.59 & 3.65 & 5.396 & 3.35 & 0.888 & 0.189 & 8.63 & 0.98 & 0.52 & 0.15 & 9.45 & 0.62 & 0.234 & 0.13 \\
\hline H19 F3 & 3.022 & 0.19 & 0.148 & 0.013 & 1.378 & 0.071 & 20.84 & 0.61 & 8.5 & 0.38 & 3.946 & 0.70 & 0.958 & 0.046 & 13.08 & 0.52 & & 0.04 & 46.63 & 0.66 & 0.402 & 0.03 \\
\hline $\mathrm{H} 20 \mathrm{~F} 1$ & 3.078 & 0.28 & 0.17 & 0.023 & 1.348 & 0.104 & 27.48 & 2.31 & 12.46 & 0.65 & 7.496 & 1.90 & 0.824 & 0.061 & 12.73 & 0.79 & 0.688 & 0.10 & 33.24 & 4.03 & 0.39 & 0.07 \\
\hline $\mathrm{H} 20 \mathrm{~F} 2$ & 3.134 & 0.25 & 0.178 & 0.026 & 1.336 & 0.156 & 26.01 & 6.37 & 12.82 & 2.14 & 7.602 & 1.84 & 0.832 & 0.085 & 11.68 & 0.93 & 0.714 & 0.10 & 35.12 & 7.55 & 0.412 & 0.16 \\
\hline H20 F3 & 3.03 & 0.29 & 0.168 & 0.024 & 1.33 & 0.100 & 24.95 & 1.39 & 12.73 & 2.59 & 7.248 & 1.82 & 0.834 & 0.054 & 12.46 & 0.92 & 0.722 & 0.09 & 36.02 & 3.39 & 0.414 & 0.06 \\
\hline $\mathrm{H} 21 \mathrm{~F} 1$ & 4.468 & 0.33 & 0.196 & 0.015 & 1.508 & 0.056 & 42.21 & 3.82 & 13.85 & 0.41 & 7.658 & 0.64 & 0.742 & 0.030 & 12.1 & 0.79 & 0.43 & 0.08 & 16.52 & 4.94 & 0.198 & 0.10 \\
\hline H21 F2 & 4.09 & 0.57 & 0.148 & 0.069 & 1.486 & 0.146 & 35.22 & 6.54 & 13.56 & 1.52 & 6.882 & 1.65 & 0.79 & 0.075 & 11.91 & 2.90 & 0.552 & 0.17 & 25.04 & 6.82 & 0.262 & 0.11 \\
\hline H21 F3 & 4.476 & 0.31 & 0.196 & 0.015 & 1.55 & 0.032 & 42.36 & 4.40 & 14.29 & 1.11 & 7.33 & 0.62 & 0.766 & 0.078 & 11.34 & 0.26 & 0.466 & 0.15 & 16.96 & 6.21 & 0.162 & 0.05 \\
\hline $\mathrm{H} 22 \mathrm{~F} 1$ & 4.432 & 0.41 & 0.194 & 0.018 & 1.514 & 0.062 & 42.9 & 6.59 & 14.23 & 0.49 & 7.238 & 0.76 & 0.752 & 0.055 & 10.27 & 1.72 & 0.44 & 0.12 & 17.72 & 7.41 & 0.182 & 0.07 \\
\hline $\mathrm{H} 22 \mathrm{~F} 2$ & 4.28 & 0.66 & 0.19 & 0.023 & 1.482 & 0.215 & 38.87 & 6.26 & 13.74 & 0.73 & 7.504 & 1.59 & 0.764 & 0.074 & 10.78 & 2.91 & 0.558 & 0.16 & 21.57 & 5.32 & 0.194 & 0.03 \\
\hline H22 F3 & 4.388 & 0.39 & 0.24 & 0.096 & 1.268 & 0.603 & 41.57 & 5.31 & 13.38 & 1.25 & 8.304 & 2.68 & 0.788 & 0.048 & 11.86 & 0.71 & 0.464 & 0.08 & 17.38 & 7.11 & 0.234 & 0.10 \\
\hline ANOVA $F$ & 17.27 & & 5.15 & & 11.51 & & 54.03 & & 14.55 & & 15.47 & & 6.32 & & 48 & & 33.04 & & 56 & & 38.81 & \\
\hline $\mathrm{LSD}_{0.001}$ & 0.9 & & 0.1 & & 0.4 & & 8.15 & & 3.14 & & 2.939 & & 0.2 & & 2.51 & & 0.2 & & 7.91 & & 0.2 & \\
\hline
\end{tabular}




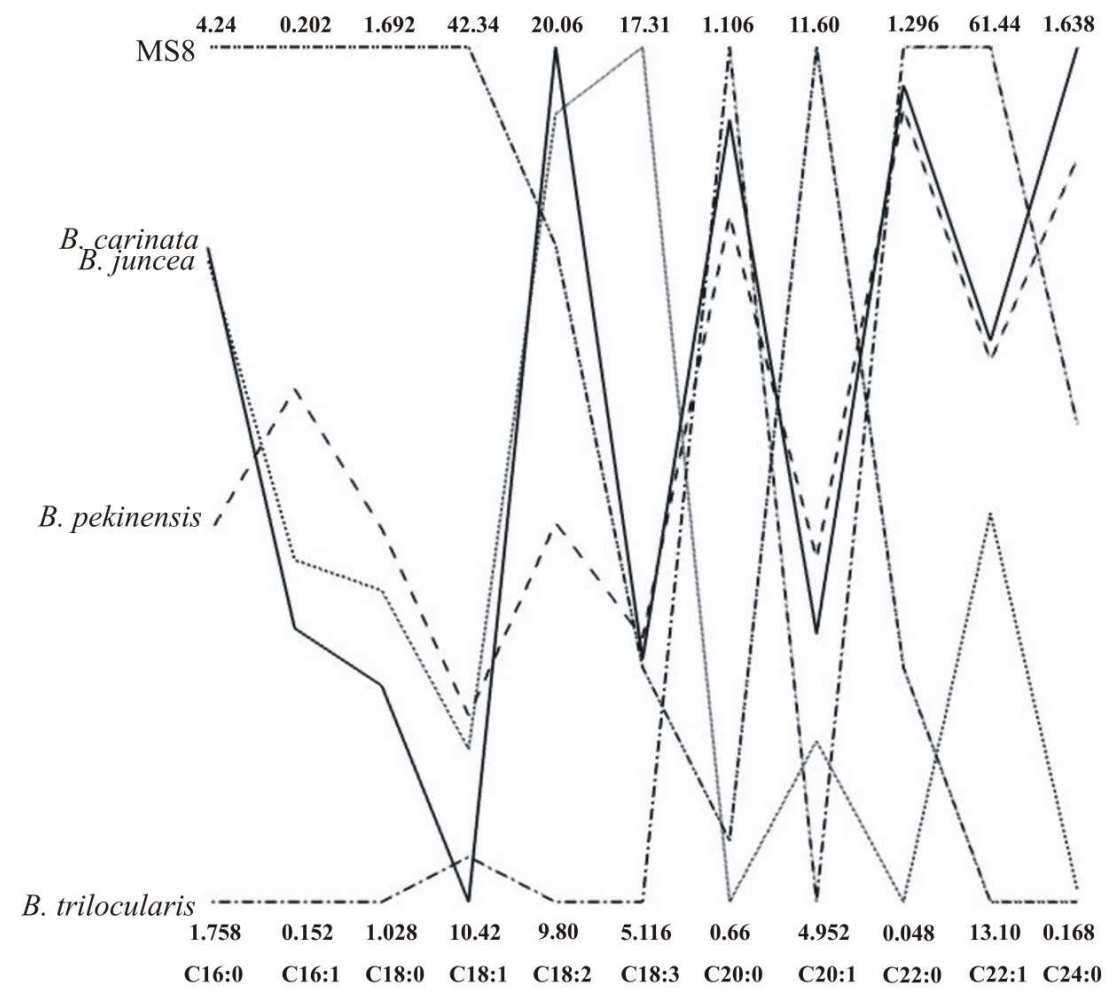

Figure 1. Parallel coordinate plots (PCPs) for five parental forms and 11 traits.

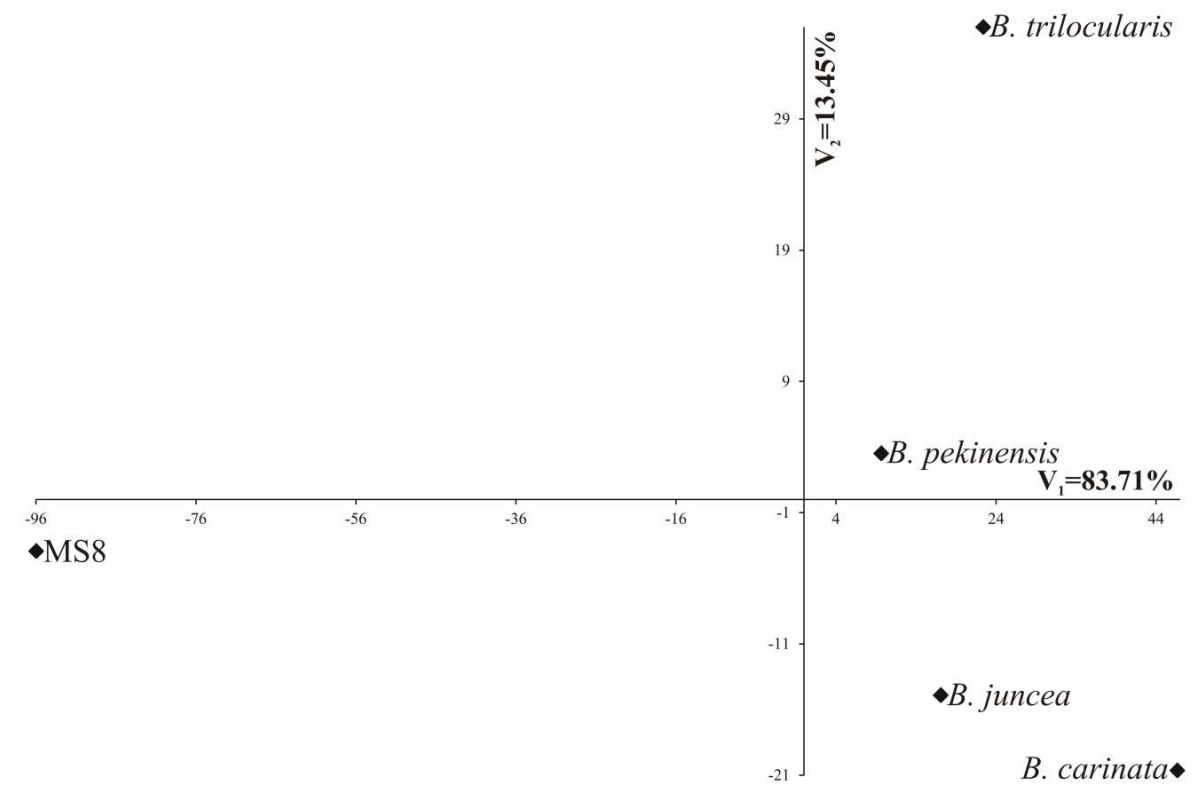

Figure 2. Location of Brassica parental forms in the system of the first two canonical variables.

Table 4. Mahalanobis distances for parental forms.

\begin{tabular}{cccccc}
\hline Parental Form & MS8 & B. carinata & B. juncea & B.pekinensis & B. trilocularis \\
\hline MS8 & - & & & \\
B. carinata & 143.8 & - & - & \\
B. juncea & 115.0 & 39.4 & 30.4 & - & - \\
B. pekinensis & 106.1 & 44.7 & 53.2 & 36.3 & - \\
B. trilocularis & 125.0 & 62.6 &
\end{tabular}


Generally, 458 significant heterosis effects were obtained: 148 in $\mathrm{F}_{1}, 151$ in $\mathrm{F}_{2}$ and 159 in $\mathrm{F}_{3}$ (Table 5). The number of the significant heterosis effects for individual hybrids ranged from eight (for H18) to 28 (for H1) (Table 5). Considering observed traits, the lowest number of statistically significant heterosis effects was obtained for C16:1 (14), however, the highest was for C22:0 and C24:0 (62) (Table 3). The obtained results indicated statistically significant heterosis effects in all three generations for 122 out of 242 cases. In 61 cases, we observed non-significant heterosis effects in each of the three generations. Statistically significant heterosis effects for individual hybrid had the same sign in particular generations, except H6 for C22:0, H11 for C20:1, H14 for C16:0, C18:1, C22:0 and C22:1, as well as H19 for C18:1 and C22:1 (Table 4). The hybrid H5 is recommended for further inclusion in the breeding programs because it has the highest positively heterosis effects for $\mathrm{C} 18: 1$, and negatively for C18:2, C18:3 and C22:1 in all three generations.

Table 5. Heterosis effects for particular hybrids in three generations.

\begin{tabular}{|c|c|c|c|c|c|c|c|c|c|c|c|}
\hline Hybrid & C16:0 & C16:1 & C18:0 & C18:1 & C18:2 & C18:3 & C20:0 & C20:1 & C22:0 & C22:1 & C24:0 \\
\hline H1 F1 & $1 * *$ & 0.03 & 0.2 & $13^{* * *}$ & $-3.98^{* * *}$ & -1.272 & $-0.1 *$ & $3.9^{* * *}$ & $-0.4^{* * *}$ & $-11^{* * *}$ & $-0.7^{* * *}$ \\
\hline H1 F2 & $1 *$ & $0.25^{* * *}$ & $0.3^{* *}$ & $11^{* * *}$ & $-3.09^{* * *}$ & -0.17 & $-0.5^{* * *}$ & $3.5^{* * *}$ & $-0.7^{* * *}$ & $-10 * * *$ & $-0.8^{* * *}$ \\
\hline H1 F3 & $1 *$ & $0.14^{* * *}$ & $0.2 *$ & $12^{* * *}$ & $-3.53^{* * *}$ & -0.721 & $-0.3^{* * *}$ & $3.7^{* * *}$ & $-0.6^{* * *}$ & $-11^{* * *}$ & $-0.7^{* * *}$ \\
\hline H2 F1 & $1 *$ & 0 & 0.2 & $4^{*}$ & $-8.49^{* * *}$ & $-4.21^{* * *}$ & 0 & $6.9^{* * *}$ & $-0.2^{* * *}$ & 1 & $-0.6^{* * *}$ \\
\hline $\mathrm{H} 2 \mathrm{~F} 2$ & $1 *$ & 0.02 & $0.2 *$ & $4^{*}$ & $-7.05^{* * *}$ & $-4.574^{* * *}$ & 0 & $6.7^{* * *}$ & $-0.2^{* * *}$ & 0 & $-0.7^{* * *}$ \\
\hline $\mathrm{H} 2 \mathrm{~F} 3$ & $1 *$ & 0.01 & $0.2 *$ & $4 *$ & $-7.84^{* * *}$ & $-4.385^{* * *}$ & 0 & $6.9^{* * *}$ & $-0.2^{* * *}$ & 1 & $-0.7^{* * *}$ \\
\hline H3 F1 & 0 & 0 & 0 & $7^{* *}$ & $-5.3^{* * *}$ & $-1.994^{*}$ & $-0.1 *$ & $6.4^{* * *}$ & $-0.4^{* * *}$ & $-5^{* *}$ & $-0.7^{* * *}$ \\
\hline H3 F2 & 0 & $-0.06 *$ & 0.1 & $7^{* *}$ & $-5.41^{* * *}$ & $-2.146^{* *}$ & 0.1 & $6.7^{* * *}$ & $-0.4^{* * *}$ & $-6^{* *}$ & $-0.7^{* * *}$ \\
\hline H3 F3 & 0 & -0.03 & 0.1 & $7^{* *}$ & $-5.36^{* * *}$ & $-2.01^{* *}$ & 0 & $6.5^{* * *}$ & $-0.4^{* * *}$ & $-5^{* *}$ & $-0.7^{* * *}$ \\
\hline H4 F1 & $1 *$ & 0.01 & 0.2 & $10^{* * *}$ & $-5.03^{* * *}$ & $-1.852^{*}$ & $-0.1 *$ & $5.1^{* * *}$ & $-0.4^{* * *}$ & $-8^{* * *}$ & $-0.7^{* * *}$ \\
\hline H4 F2 & $1 *$ & -0.04 & 0.1 & $9 * * *$ & $-5.13^{* * *}$ & $-1.886^{*}$ & 0.1 & $5.9^{* * *}$ & $-0.4^{* * *}$ & $-8^{* * *}$ & $-0.7^{* * *}$ \\
\hline H4 F3 & $1 *$ & -0.01 & 0.2 & $9 * * *$ & $-5.09 * * *$ & $-1.88 *$ & 0 & $5.7^{* * *}$ & $-0.4^{* * *}$ & $-8^{* * *}$ & $-0.7^{* * *}$ \\
\hline H5 F1 & $2^{* * *}$ & $0.06^{*}$ & $0.7^{* * *}$ & $33^{* * *}$ & $-8.7^{* * *}$ & $-5.136^{* * *}$ & 0 & -0.5 & $-0.4^{* * *}$ & $-21^{* * *}$ & $-0.7^{* * *}$ \\
\hline H5 F2 & $2 * * *$ & 0.05 & $1.2^{* * *}$ & $33^{* * *}$ & $-8.46^{* * *}$ & $-5.124^{* * *}$ & -0.1 & -0.7 & $-0.4^{* * *}$ & $-21 * * *$ & $-0.7^{* * *}$ \\
\hline H5 F3 & $2 * * *$ & $0.06^{*}$ & $0.9^{* * *}$ & $33^{* * *}$ & $-8.77^{* * *}$ & $-5.124^{* * *}$ & -0.1 & -0.6 & $-0.4^{* * *}$ & $-21^{* * *}$ & $-0.7^{* * *}$ \\
\hline H6 F1 & 0 & -0.02 & $-0.2 *$ & $-10 * * *$ & $-5.37 * * *$ & 0.843 & 0 & $-2.3^{* * *}$ & $-0.2^{* * *}$ & $17^{* * *}$ & 0 \\
\hline H6 F2 & 0 & $0.09^{* * *}$ & -0.2 & $-12 * * *$ & $-4.55^{* * *}$ & 0.187 & $0.7^{* * *}$ & $-1.5^{*}$ & $0.2^{* * *}$ & $17^{* * *}$ & $0.7^{* * *}$ \\
\hline H6 F3 & 0 & 0.04 & -0.2 & $-12 * * *$ & $-4.89^{* * *}$ & 1.511 * & $0.4^{* * *}$ & $-2 * *$ & 0 & $17^{* * *}$ & $0.4^{* * *}$ \\
\hline H7 F1 & 0 & 0.04 & 0.1 & $32 * * *$ & -0.11 & $-3.865^{* * *}$ & 0 & $-6^{* * *}$ & $0.2^{* * *}$ & $-23^{* * *}$ & -0.1 \\
\hline H7 F2 & 0 & $0.15^{* * *}$ & $0.3^{* *}$ & $31 * * *$ & -0.37 & $-3.961^{* * *}$ & $0.2^{* *}$ & $-5.1^{* * *}$ & $0.2^{* * *}$ & $-23 * * *$ & 0 \\
\hline H7 F3 & 0 & $0.18^{* * *}$ & $0.3^{*}$ & $23^{* * *}$ & -1.16 & $-2.964^{* * *}$ & $0.2^{* *}$ & $-4.8^{* * *}$ & $0.2^{* * *}$ & $-15^{* * *}$ & 0 \\
\hline H8 F1 & $1 *$ & 0 & $0.3^{* *}$ & $10^{* * *}$ & -1.1 & -1.13 & -0.1 & $4.1^{* * *}$ & $-0.4^{* * *}$ & $-12 * * *$ & $-0.8^{* * *}$ \\
\hline H8 F2 & 0 & 0 & $0.2 *$ & $10^{* * *}$ & $-1.7^{*}$ & -1.16 & -0.1 & $5.2^{* * *}$ & $-0.4^{* * *}$ & $-12 * * *$ & $-0.7^{* * *}$ \\
\hline H8 F3 & 0 & 0 & 0.1 & $7 * * *$ & -0.73 & -0.416 & -0.1 & $4.4^{* * *}$ & $-0.4^{* * *}$ & $-10 * * *$ & $-0.6^{* * *}$ \\
\hline H9 F1 & 0 & 0 & 0.1 & -3 & $-3.15^{* * *}$ & -1.384 & 0 & $6.6^{* * *}$ & $-0.4^{* * *}$ & 2 & $-0.6^{* * *}$ \\
\hline H9 F2 & $1^{* *}$ & $0.05^{*}$ & 0 & 2 & -0.97 & $-1.752 *$ & -0.1 & $4.5^{* * *}$ & $-0.2^{* * *}$ & -4 & $-0.6^{* * *}$ \\
\hline H9 F3 & $1 * * *$ & 0.02 & $0.3^{*}$ & 2 & $-4.92^{* * *}$ & $-4.032^{* * *}$ & 0 & $7.1^{* * *}$ & $-0.3^{* * *}$ & -1 & $-0.6^{* * *}$ \\
\hline H10 F1 & 0 & -0.02 & 0 & -1 & $-4.75^{* * *}$ & $-3.17^{* * *}$ & 0 & $4^{* * *}$ & $-0.1^{* *}$ & $5 *$ & $-0.4^{* * *}$ \\
\hline H10 F2 & $1 * * *$ & 0.02 & $0.2 *$ & 2 & $-4.56^{* * *}$ & $-3.838^{* * *}$ & 0 & $4.3^{* * *}$ & $-0.3^{* * *}$ & 1 & $-0.5^{* * *}$ \\
\hline H10 F3 & $2 * * *$ & 0.03 & $0.4^{* * *}$ & $5 *$ & $-5.23^{* * *}$ & $-2.864^{* * *}$ & 0.1 & $5 * * *$ & $-0.3^{* * *}$ & -4 & $-0.6^{* * *}$ \\
\hline H11 F1 & $1 * *$ & 0 & $0.3^{* * *}$ & $17^{* * *}$ & -0.85 & -0.876 & -0.1 & $2 * *$ & $-0.4^{* * *}$ & $-17 * * *$ & $-0.7^{* * *}$ \\
\hline H11 F2 & $1 * * *$ & 0.02 & $0.4^{* * *}$ & $28^{* * *}$ & 1.46 & -0.956 & -0.1 & $-4.5^{* * *}$ & $-0.4^{* * *}$ & $-24^{* * *}$ & $-0.8^{* * *}$ \\
\hline H11 F3 & $1^{* * *}$ & 0.01 & $0.4^{* * *}$ & $31^{* * *}$ & $3.8^{* * *}$ & -0.412 & -0.1 & $-7.3^{* * *}$ & $-0.4^{* * *}$ & $-27 * * *$ & $-0.8^{* * *}$ \\
\hline H12 F1 & $1^{*}$ & 0.01 & 0.2 & $10^{* * *}$ & 0.47 & 0.574 & $-0.1^{*}$ & $2.7^{* * *}$ & $-0.4^{* * *}$ & $-13^{* * *}$ & $-0.7^{* * *}$ \\
\hline H12 F2 & $1 * *$ & 0.01 & $0.5^{* * *}$ & $18^{* * *}$ & -0.71 & -1.506 & -0.1 & 1.2 & $-0.4^{* * *}$ & $-17 * * *$ & $-0.7^{* * *}$ \\
\hline H12 F3 & 0 & 0 & $0.3^{* *}$ & $12^{* * *}$ & -1.29 & -0.714 & -0.1 & $3.3^{* * *}$ & $-0.4^{* * *}$ & $-13^{* * *}$ & $-0.7^{* * *}$ \\
\hline H13 F1 & $1^{* * *}$ & $0.08^{* *}$ & $0.3 *$ & $16^{* * *}$ & $-2.98^{* * *}$ & $-2.62^{* * *}$ & 0 & $2.9^{* * *}$ & $-0.3^{* * *}$ & $-15^{* * *}$ & $-0.6^{* * *}$ \\
\hline H13 F2 & $2 * * *$ & 0.05 & $0.6^{* * *}$ & $23^{* * *}$ & $-3.04^{* * *}$ & $-2.956^{* * *}$ & 0 & -0.3 & $-0.3^{* * *}$ & $-19 * * *$ & $-0.6^{* * *}$ \\
\hline H13 F3 & $3^{* * *}$ & $0.06^{*}$ & $0.7^{* * *}$ & $29^{* * *}$ & $-6.21^{* * *}$ & $-5.78^{* * *}$ & 0.1 & -0.9 & $-0.2^{* * *}$ & $-19 * * *$ & $-0.6^{* * *}$ \\
\hline H14 F1 & $-1^{* *}$ & -0.05 & -0.2 & $-11^{* * *}$ & $-8.2^{* * *}$ & $-4.77^{* * *}$ & $0.1^{*}$ & $2.6^{* * *}$ & $0.2^{* * *}$ & $22 * * *$ & $-0.5^{* * *}$ \\
\hline H14 F2 & 0 & -0.03 & 0.1 & 1 & $-2.83^{* * *}$ & -0.832 & 0 & $6.4^{* * *}$ & $-0.4^{* * *}$ & -3 & $-0.6^{* * *}$ \\
\hline H14 F3 & $1^{* *}$ & $0.07^{*}$ & 0.1 & $5 *$ & $-2.74^{* * *}$ & $-2.066^{* *}$ & 0.1 & $5.7^{* * *}$ & $-0.3^{* * *}$ & $-6^{* *}$ & $-0.7^{* * *}$ \\
\hline H15 F1 & $1^{* * *}$ & 0.02 & $0.3^{* * *}$ & $7^{* *}$ & $-4.95^{* * *}$ & $-4.168^{* * *}$ & 0 & $6.4^{* * *}$ & $-0.3^{* * *}$ & $-5^{*}$ & $-0.6^{* * *}$ \\
\hline H15 F2 & $1^{* * *}$ & 0.02 & $0.4^{* * *}$ & $7^{* * *}$ & $-3.57^{* * *}$ & $-2.43^{* *}$ & 0 & $5 * * *$ & $-0.4^{* * *}$ & $-7^{* * *}$ & $-0.6^{* * *}$ \\
\hline H15 F3 & $1^{* * *}$ & 0.02 & $0.3^{*}$ & $8^{* * *}$ & $-3.33^{* * *}$ & $-2.486^{* *}$ & -0.1 & $5.1^{* * *}$ & $-0.4^{* * *}$ & $-8^{* * *}$ & $-0.6^{* * *}$ \\
\hline H16 F1 & $1^{* *}$ & 0.04 & 0 & 3 & -0.21 & -0.15 & -0.1 & $4.1^{* * *}$ & $-0.4^{* * *}$ & $-7^{* *}$ & $-0.6^{* * *}$ \\
\hline H16 F2 & $1^{* *}$ & 0.03 & $0.2 *$ & $6^{* *}$ & 0.24 & -0.606 & -0.1 & $3.6^{* * *}$ & $-0.4^{* * *}$ & $-10 * * *$ & $-0.6^{* * *}$ \\
\hline H16 F3 & $1 * * *$ & 0.03 & 0.2 & $11^{* * *}$ & -0.98 & $-2.038^{* *}$ & -0.1 & $3.5^{* * *}$ & $-0.4^{* * *}$ & $-12 * * *$ & $-0.7^{* * *}$ \\
\hline
\end{tabular}


Table 5. Cont.

\begin{tabular}{|c|c|c|c|c|c|c|c|c|c|c|c|}
\hline Hybrid & C16:0 & C16:1 & C18:0 & C18:1 & C18:2 & C18:3 & C20:0 & C20:1 & C22:0 & C22:1 & C24:0 \\
\hline H17 F1 & $2^{* * *}$ & 0.02 & 0.2 & $16^{* * *}$ & 0.99 & 0.55 & $-0.1^{*}$ & $2.6^{* * *}$ & $-0.4^{* * *}$ & $-21^{* * *}$ & $-0.4^{* * *}$ \\
\hline H17 F2 & $1^{* * *}$ & -0.02 & 0.1 & $9^{* * *}$ & 0.86 & 0.434 & $-0.1^{*}$ & $2.6^{* * *}$ & $-0.3^{* * *}$ & $-14^{* * *}$ & $-0.3^{* * *}$ \\
\hline H17 F3 & $2^{* * *}$ & 0.02 & $0.2 *$ & $16^{* * *}$ & 0.21 & 0.002 & -0.1 & $2.7^{* * *}$ & $-0.4^{* * *}$ & $-21^{* * *}$ & $-0.4^{* * *}$ \\
\hline H18 F1 & 0 & 0 & 0 & -2 & -0.6 & 0.782 & -0.1 & $4.2 * * *$ & $-0.1^{* *}$ & -2 & $-0.2 * *$ \\
\hline H18 F2 & 0 & 0 & -0.1 & -3 & -1.25 & 0.686 & -0.1 & $3.7^{* * *}$ & -0.1 & 0 & $-0.1^{* *}$ \\
\hline H18 F3 & 0 & 0 & 0 & -2 & -0.52 & 0.852 & -0.1 & $4.2^{* * *}$ & $-0.1^{* *}$ & -3 & $-0.2^{* * *}$ \\
\hline H19 F1 & 0 & -0.02 & 0.1 & -4 & $-4.48^{* * *}$ & $-2.392^{* *}$ & 0 & $4.5^{* * *}$ & 0 & $6^{* *}$ & $-0.2^{* * *}$ \\
\hline H19 F2 & $3^{* * *}$ & $0.07^{*}$ & $0.6^{* * *}$ & $27^{* * *}$ & -1.15 & -1.392 & 0 & 0.4 & $-0.3^{* * *}$ & $-28^{* * *}$ & $-0.3^{* * *}$ \\
\hline H19 F3 & 0 & -0.03 & 0 & $-6^{* *}$ & $-5.24^{* * *}$ & $-2.842^{* * *}$ & 0.1 & $4.8^{* * *}$ & 0.1 & $9^{* * *}$ & $-0.2^{* * *}$ \\
\hline H20 F1 & 0 & -0.01 & 0 & 0 & -1.28 & 0.708 & -0.1 & $4.5^{* * *}$ & $-0.2 * *$ & -4 & $-0.2^{* * *}$ \\
\hline H20 F2 & 0 & 0 & 0 & -1 & -0.91 & 0.814 & -0.1 & $3.4^{* * *}$ & $-0.1^{* *}$ & -2 & $-0.2^{* *}$ \\
\hline H20 F3 & 0 & -0.01 & 0 & -2 & -1.01 & 0.46 & -0.1 & $4.2^{* * *}$ & $-0.1 *$ & -1 & $-0.2^{* *}$ \\
\hline H21 F1 & $1^{* * *}$ & 0.02 & 0.1 & $15^{* * *}$ & 0.12 & 0.87 & $-0.2 *$ & $3.8^{* * *}$ & $-0.4^{* * *}$ & $-21^{* * *}$ & $-0.4^{* * *}$ \\
\hline H21 F2 & $1^{* * *}$ & -0.03 & 0.1 & $8^{* * *}$ & -0.18 & 0.094 & -0.1 & $3.6^{* * *}$ & $-0.3^{* * *}$ & $-12^{* * *}$ & $-0.3^{* * *}$ \\
\hline H21 F3 & $1^{* * *}$ & 0.02 & 0.2 & $15^{* * *}$ & 0.56 & 0.542 & $-0.1^{*}$ & $3.1^{* * *}$ & $-0.4^{* * *}$ & $-20^{* * *}$ & $-0.4^{* * *}$ \\
\hline H22 F1 & $1^{* * *}$ & 0.02 & 0.2 & $16^{* * *}$ & 0.49 & 0.45 & $-0.1 *$ & $2 * *$ & $-0.4^{* * *}$ & $-20 * * *$ & $-0.4^{* * *}$ \\
\hline $\mathrm{H} 22$ F2 & $1^{* * *}$ & 0.01 & 0.1 & $12 * * *$ & 0 & 0.716 & $-0.1 *$ & $2.5^{* * *}$ & $-0.3^{* * *}$ & $-16^{* * *}$ & $-0.4^{* * *}$ \\
\hline H22 F3 & $1^{* * *}$ & $0.06^{*}$ & -0.1 & $14^{* * *}$ & -0.36 & 1.516 * & -0.1 & $3.6^{* * *}$ & $-0.4^{* * *}$ & $-20^{* * *}$ & $-0.3^{* * *}$ \\
\hline
\end{tabular}

Individual traits are of different importance and have a different share in the joint multivariate variation. A study on the multivariate variation for treatments also includes identification of the most important traits in the multivariate variation of treatments. Canonical variables analysis (CVA) is a statistical tool making it possible to solve this problem $[27,28]$. The results of the analysis of canonical variables for investigated hybrids analyzed in three years are presented in Figure 3 . The first two canonical variables explained jointly $66.37 \%$ total variation between hybrids. The first canonical variable accounted for $38.97 \%$ of the total variation, while the second canonical variable accounted for $27.40 \%$ (Figure 3 ).

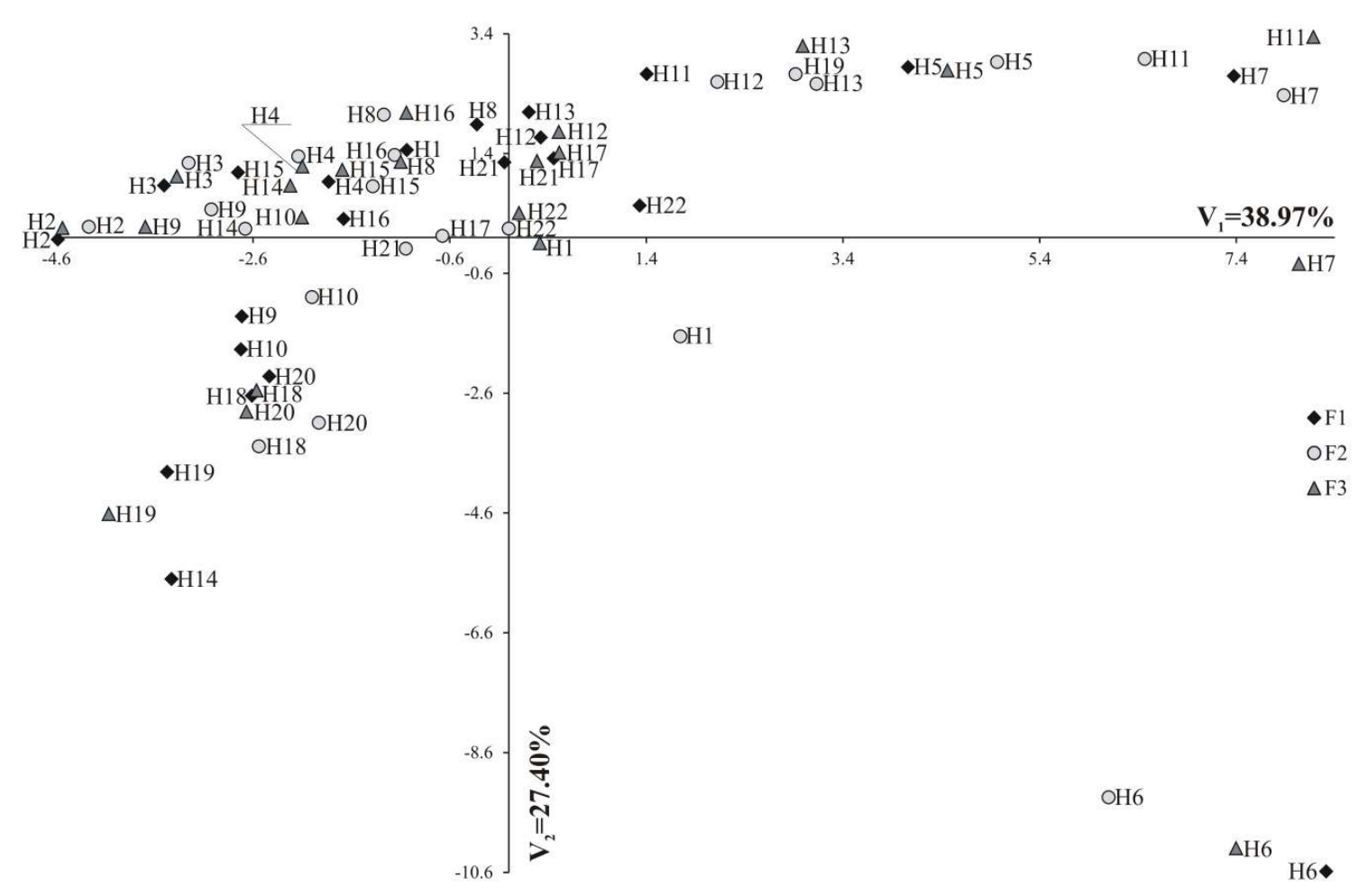

Figure 3. Location of Brassica hybrids in the system of the first two canonical variables. 
The most significant, positive, linear relationship between the first canonical variables was found for C16:0, C16:1, C18:0, C18:1, C18:2 and C18:3, while it was negative for C20:1, C22:0 and C22:1. The second canonical variable was significantly positively correlated with C16:0,18:1 and C18:2, while it was negatively correlated with C18:3, C20:0, C22:1 and C24:0. The greatest variation in terms of all the 11 fatty acids jointly (measured Mahalanobis distances) was found for $\mathrm{H} 3$ in $\mathrm{F}_{2}$ and $\mathrm{H} 6$ in $\mathrm{F}_{1}$ (the Mahalanobis distance between them amounted to 19.919). The greatest similarity was found for $\mathrm{H} 18$ in $\mathrm{F}_{1}$ and $\mathrm{H} 18$ in $\mathrm{F}_{3}$ (0.150). According to the literature data, canonical variable analysis and Mahalanobis distances are statistical tools, which may be confirmed by their extensive application by breeders and geneticists [29-34].

Our results fully confirmed that assumption and proved that in order to identify the best Brassica genotypes in respect of heterosis for fatty acids requirements, the multivariate analysis of variance conducted for the eleven analyzed acids is a very useful statistical method of high importance.

Some authors reported that, most probably, it will soon be possible to change the profile of fatty acids content in Brassica seed oil, according to industry expectations [35,36].

Moreover, interspecific hybridization between appropriate Brassica species have a great potential in creating lines with a modified fatty acid composition [37,38]. In particular, as our results showed, the development of lines with a high oleic acid content seems promising. According to the magnitude of the predicted breeding values in this study, it is possible to select desirable breeding materials (hybrid line H5), which show the proper spectrum of the fatty acids in oil.

\section{Conclusions}

All analyzed parental genotypes as well as Brassica hybrid lines show statistically significant multivariate diversity for eleven fatty acids. The greatest variation of parental forms in terms of all the traits jointly was found for B. napus MS8 line and B. carinata. These two genotypes would be the best as parental forms in future breeding programs.

Analysis of canonical variables is a good tool for multivariate analysis of relationships among genotypes. The first two canonical variables explained jointly $97.16 \%$ and $66.37 \%$ total variation between parental forms and hybrids, respectively.

The highest number of significant heterosis effects was observed for behenic and lignoceric acids and for Brassica hybrid line H1.

The hybrid line $\mathrm{H} 5$ is recommended for further inclusion in the breeding programs, because it has the highest positively heterosis effect for oleic acid, and negatively for linoleic, linolenic and erucic acids in all three generations.

Author Contributions: Conceptualization, J.N. and J.B.; methodology, J.N. and K.S.-S.; software, J.B.; validation, J.N., J.B., K.S.-S. and T.W.; formal analysis, J.N., J.B. and K.S.-S.; investigation, J.N., J.B. and T.W.; data curation, J.N., J.B. and T.W.; writing-original draft preparation, J.N.; writing-review and editing, J.N., J.B. and T.W.; visualization, J.N., J.B. and T.W.; supervision, J.N., J.B.; project administration, J.N.; funding acquisition, J.N. All authors have read and agreed to the published version of the manuscript.

Funding: This research was funded by the Polish Ministry of Science and Higher Education, project number N N310 308234.

Acknowledgments: The publication was co-financed within the framework of the Ministry of Science and Higher Education program titled "Regional Initiative Excellence" in 2019-2022, project no. 005/RID/2018/19.

Conflicts of Interest: The authors declare no conflict of interest.

\section{References}

1. Schuster, W.; Michael, J. Untersuchungen über Inzuchtdepressionen und Heterosis effekte bei Raps (Brassica napus oleifera). Z Pflanz. 1976, 77, 55-66.

2. Sernyk, J.L.; Stefansson, B.R. Heterosis in summer rape. Can. J. Plant Sci. 1983, 63, 407-414. [CrossRef] 
3. Chen, B.Y.; Heneen, W.K. Fatty acid composition of resynthesized Brassica napus L., B. campestris L. and B. alboglabra Bailey with special reference to the inheritance of erucic acid content. Heredity 1989, 63, 309-314. [CrossRef]

4. Singh, S.; Dey, S.S.; Bhatia, R.; Kumar, R.; Sharma, K.; Behera, T.K. Heterosis and combining ability in cytoplasmic male sterile and doubled haploid based Brassica oleracea progenies and prediction of heterosis using microsatellites. PLoS ONE 2019, 14, e0210772. [CrossRef] [PubMed]

5. Fick, G.N. Genetics and breeding of sunflower. JAOCS 1983, 60, 1252-1253. [CrossRef]

6. Friedt, W. Biotechnology in breeding of industrial oil crops-The present status and future prospects. Fur Sci. Technol. 1988, 90, 51-55. [CrossRef]

7. Chen, B.Y.; Gertsson, B. Genotypes for high oleic acid content (about 80\%) in the oil of rapeseed (Brassica napus L.). EUCARPIA Crucif. Newsl. 1988, 13, 46-47.

8. Simopoulos, A. The importance of the omega-6/omega-3 fatty acid ratio in cardiovascular disease and other chronic diseases. Exp. Biol. Med. 2008, 233, 674-688. [CrossRef] [PubMed]

9. Ramos, M.J.; Fernández, C.M.; Casas, A.; Rodríguez, L.; Pérez, A. Influence of fatty acid composition of raw materials on biodiesel properties. Bioresour. Technol. 2009, 100, 261-268. [CrossRef] [PubMed]

10. Priyamedha, S.; Singh, B.K.; Ram, B.; Kumar, A.; Singh, V.V.; Meena, M.L.; Singh, D. Development and Evaluation of Double Low Quality Lines of Indian mustard (Brassica juncea L. Czern \&Coss). SABRAO J. Breed Genet. 2014, 46, 274-283.

11. Ali, N.; Bakht, J.; Naveed, K.; Liaquat, M.; Khan, S.A.; Saeed, M.; Ali, S.; Hussain, I.; Khan, S.M.; Salim, M. Heterosis studies for some fatty acids composition of Indian Mustard (Brassica juncea L.). J. Anim. Plant Sci. 2015, 25, 587-592.

12. Hassan, M.F.; Seyis, F.; Badani, A.G.; Pons-Kühnemann, J.; Friedt, W.; Lühs, W.; Snowdon, R.J. Surveying genetic diversity in the Brassica napus L. gene pool using SSR markers. Genet. Resour. Crop Evol. 2006, 53, 793-802. [CrossRef]

13. Pal, B.P.; Sikka, S.M. Exploitation of hybrid vigour in the improvement of crop plants fruits and vegetables. Indian J. Genet. Plant Breed. 1956, 16, 95-193.

14. Niemann, J.; Bocianowski, J.; Wojciechowski, A. Effects of genotype and environment on seed quality traits variability in interspecific cross-derived Brassica lines. Euphytica 2018, 214, 193. [CrossRef]

15. Momotaz, A.; Kato, M.; Kakihara, F. Variation in seedfertility and fatty acid composition in allohexaploids between Brassica carinata and Sinapis species with the advance of generations. Breed. Sci. 2000, 2, 91-100. [CrossRef]

16. Shapiro, S.S.; Wilk, M.B. An analysis of variance test for normality (complete samples). Biometrika 1965, 52, 591-611. [CrossRef]

17. Rencher, A.C. Interpretation of canonical discriminant functions, canonical variates, and principal components. Am. Stat. 1992, 46, 217-225.

18. Mahalanobis, P.C. On the generalized distance in statistics. Proc. Natl. Acad. Sci. India A 1936, 12, 49-55.

19. Seidler-Łożykowska, K.; Bocianowski, J. Evaluation of variability of morphological traits of selected caraway (Carum carvi L.) genotypes. Ind. Crop. Prod. 2012, 35, 140-145. [CrossRef]

20. Gehringer, A.; Snowdon, R.; Spiller, T.; Basunanda, P.; Friedt, W. New oilseed rape (Brassica napus) hybrids with high levels of heterosis for seed yield under nutrient-poor conditions. Breed. Sci. 2007, 57, 315-320. [CrossRef]

21. Qu, C.; Jia, L.; Fu, F.; Zhao, H.; Lu, K.; Wei, L.; Xu, X.; Liang, Y.; Li, S.; Wang, R.; et al. Genome-wide association mapping and identification of candidate genes for fatty acid composition in Brassica napus L. using SNP markers. BMC Genom. 2017, 18, 232. [CrossRef]

22. Cuthbert, R.D.; Crow, G.; Mcvetty, P.B.E. Assessment of seed quality performance and heterosis for seed quality traits in hybrid high erucic acid rapeseed (HEAR). Can. J. Plant Sci. 2011, 91, 837-846. [CrossRef]

23. Adamska, E.; Cegielska-Taras, T.; Kaczmarek, Z.; Szała, L. Multivariate approach to evaluating the fatty acid composition of seed oil in a doubled haploid population of winter oilseed rape (Brassica napus L.). J. Appl. Genet. 2004, 45, 419-425. [PubMed]

24. Bocianowski, J.; Majchrzak, L. Analysis of effects of cover crop and tillage method combinations on the phenotypic traits of spring wheat (Triticum aestivum L.) using multivariate methods. Appl. Ecol. Env. Res. 2019, 17, 15267-15276. [CrossRef] 
25. Rybiński, W.; Banda, M.; Bocianowski, J.; Starzycka-Korbas, E.; Starzycki, M.; Nowosad, K. Estimation of the physicochemical variation of chickpea seeds (Cicer arietinum L.). Int. Agrophys. 2019, 33, 67-80. [CrossRef]

26. Wrońska-Pilarek, D.; Jagodziński, A.M.; Bocianowski, J.; Marecik, M.; Janyszek-Sołtysiak, M. Pollen morphology and variability of Sambucus nigra L.-Adoxaceae. Biologia 2020, 75, 481-493. [CrossRef]

27. Lahuta, L.B.; Ciak, M.; Rybiński, W.; Bocianowski, J.; Börner, A. Diversity of the composition and content of soluble carbohydrates in seeds of the genus Vicia (Leguminosae). Genet. Resour. Crop Evol. 2018, 65, 541-554. [CrossRef]

28. Radkowski, A.; Radkowska, I.; Bocianowski, J. Effect of fertilization of meadow sward with amino acids obtained from enzymatic hydrolysis on silage quality. J. Elem. 2020, 25, 259-277. [CrossRef]

29. Shamsuddin, A.K.M. Genetic diversity in relation to heterosis and combining ability in spring wheat. Theor. Appl. Genet. 1985, 70, 306-308. [CrossRef] [PubMed]

30. Seidler-Łożykowska, K.; Bocianowski, J.; Król, D. The evaluation of the variability of morphological and chemical traits of the selected lemon balm (Melissa officinalis L.) genotypes. Ind. Crops Prod. 2013, 49, 515-520. [CrossRef]

31. Nowosad, K.; Liersch, A.; Popławska, W.; Bocianowski, J. Genotype by environment interaction for seed yield in rapeseed (Brassica napus L.) using additive main effects and multiplicative interaction model. Euphytica 2016, 208, 187-194. [CrossRef]

32. Bocianowski, J.; Szulc, P.; Nowosad, K. Soil tillage methods by years interaction for dry matter of plant yield of maize (Zea mays L.) using additive main effects and multiplicative interaction model. J. Integr. Agric. 2018, 17, 2836-2839. [CrossRef]

33. Bocianowski, J.; Nowosad, K.; Szulc, P. Soil tillage methods by years interaction for harvest index of maize (Zea mays L.) using additive main effects and multiplicative interaction model. Acta Agric. Scand. B 2019, 69, 75-81. [CrossRef]

34. Wrońska-Pilarek, D.; Szkudlarz, P.; Bocianowski, J. Systematic importance of morphological features of pollen grains of species from Erica (Ericaceae) genus. PLoS ONE 2018, 13, e0204557. [CrossRef] [PubMed]

35. Cegielska-Taras, T.; Adamska, E.; Szała, L.; Kaczmarek, Z. Estimation of the genetic parameters for fatty acid content in DH lines obtained from winter oilseed rape of F1 hybrid (DH O120 × DH C-1041). Rośliny Oleiste Oilseed Crop. 2005, 26, 11-17.

36. Liersch, A.; Bocianowski, J.; Bartkowiak-Broda, I. Fatty acid and glucosinolate level in seeds of different types of winter oilseed rape cultivars (Brassica napus L.). Commun. Biometry Crop Sci. 2013, 8, 39-47.

37. Iqbal, M.C.M.; Weerakoon, S.R.; Geethanjalie, H.D.N.; Peiris, P.K.D.; Weerasena, O.V.D.S.J. Changes in the fatty acids in seeds of interspecific hybrids between Brassica napus and Brassica juncea. Crop Pasture Sci. 2011, 62, 390-395. [CrossRef]

38. Szala, L.; Kaczmarek, Z.; Adamska, E.; Cegielska-Taras, T. Assessment of winter oilseed rape DH lines using uni- and multivariate methods of quantitative genetics and mathematical methods. Biotechnol. J. Biotechnol. Comput. Biol. Bionanotechnol. 2015, 96, 171-177. [CrossRef]

(C) 2020 by the authors. Licensee MDPI, Basel, Switzerland. This article is an open access article distributed under the terms and conditions of the Creative Commons Attribution (CC BY) license (http://creativecommons.org/licenses/by/4.0/). 